Modern Physics Letters B

Vol. 30, No. 13 (2016) 1650203 (24 pages)

(C) The Author(s)

DOI: $10.1142 /$ S0217984916502031

\title{
Numerical analysis on thermal characteristics and ice melting efficiency for microwave deicing vehicle
}

\author{
Can Wang, Bo Yang, Gangfeng Tan*, Xuexun Guo, Li Zhou and Shengguang Xiong \\ Hubei Key Laboratory of Advanced Technology for Automotive Components, \\ Wuhan University of Technology, Wuhan, Hubei 430070, China \\ Hubei Collaborative Innovation Center for Automotive Components Technology, \\ Wuhan, Hubei 430070, China \\ School of Automotive Engineering, Wuhan University of Technology, \\ Wuhan, Hubei 430070, China \\ *18995570176@189.cn
}

Received 14 November 2015

Revised 26 February 2016

Accepted 1 March 2016

Published 16 May 2016

In the high latitudes, the icy patches on the road are frequently generated and have a wide distribution, which are difficult to remove and obviously affect the normal usage of the highways, bridges and airport runways. Physical deicing, such as microwave (MW) deicing, help the ice melt completely through heating mode and then the ice layer can be swept away. Though it is no pollution and no damage to the ground, the low efficiency hinders the development of MW deicing vehicle equipped without sufficient speed. In this work, the standard evaluation of deicing is put forward firstly. The intensive MW deicing is simplified to ice melting process characterized by one-dimensional slab with uniform volumetric energy generation, which results in phase transformation and interface motion between ice and water. The heating process is split into the superposition of three parts - non-heterogeneous heating for ground without phase change, heat transfer with phase change and the heat convection between top surface of ice layer and flow air. Based on the transient heat conduction theory, a mathematical model, combining electromagnetic and two-phase thermal conduction, is proposed in this work, which is able to reveal the relationship between the deicing efficiency and ambient conditions, as well as energy generation and material parameters. Using finite difference time-domain, this comprehensive model is developed to solve the moving boundary heat transfer problem in a one-dimensional structured gird. As a result, the stimulation shows the longitudinal temperature distributions in all circumstances and quantitative validation is obtained by comparing simulated temperature distributions under different conditions. In view of the best economy and fast deicing, these analytic solutions referring to the complex influence factors of deicing efficiency demonstrate the optimal matching for the new deicing design.

Keywords: Microwave deicing; melting efficiency; phase change; finite differential method.

*Corresponding author.

This is an Open Access article published by World Scientific Publishing Company. It is distributed under the terms of the Creative Commons Attribution 4.0 (CC-BY) License. Further distribution of this work is permitted, provided the original work is properly cited. 


\section{Introduction}

The common deicing methods mainly consist of chemical deicing, artificial deicing, mechanical deicing and thermal deicing. Chemical deicing method has damage to the environment, ${ }^{1}$ the efficiency of artificial deicing method which is only applicable to the thin ice is rather low, and mechanical deicing is harmful to the structure of pavement, while the traditional thermal deicing costs too much. ${ }^{2,3}$ Microwave (MW) heating equipped with penetrating ability, which is caused by the ability of materials to absorb electromagnetic energy and convert to heat, can be applied to non-contact deicing vehicle. ${ }^{4}$ In this deicing system, pavement can be heated directly, which triggers thaw at the bottom of the ice layer. However, rapid deicing on pavement has not been implemented hitherto on account of the low efficiency of deicing which is tremendously impacted by several key factors containing pavement material, ambient temperature, thickness of ice layer and MW power.

MW heating to the pavement and ice layer is non-uniform which is due to the electromagnetic waves and the variation in dielectric, physical and thermal properties of pavement and ice layer, especially the phase change of the ice. These dynamic physical situation leads to rapid changes in the material property, which claims the nonlinear computational solution. Hence, there are few exact analytic solutions to describe it systematically. What is more, the transient heat transfer problem involving melting process generally refers to moving boundary or phase change problems. Accounting for temperature-dependent properties, phase change and material with internal heat source, it is necessary to solve the nonlinear process by coupling electromagnetic and heat transfer equations. So far, a variety of conventional numerical techniques have been developed for solving these problems, including the enthalpy, ${ }^{5}$ isotherm migration ${ }^{6}$ and coordinate transformation methods. ${ }^{7}$ Pangrle et $a l .{ }^{7}$ developed a one-dimensional model of MW thawing for study, coupled electromagnetic and heat conduction model to comprehend the thawing process. The transient heat conduction solution with fixed boundary condition and the single-phase condition has been examined by Oziksik. ${ }^{8}$ Later, MV melting studies considering two-dimensional MW thawing with a fixed grid-based effective heat-capacity method coupled with Maxwell's equations were carried out by Basak and Ayappa ${ }^{9}$ The changing electric field and material properties with time and temperature varying might contribute to an uneven absorption of MW energy and subsequent non-uniformity in heating pattern. ${ }^{10}$ Recently, Salema and Afzal ${ }^{11}$ has solved a coupled electromagnetic and heat transfer model to perform simulation based on varying specific heat values. Li and Zhang ${ }^{12}$ has developed a transient solution to reveal the parameter-dependent influence on the deicing process through a carbon nanofiber polymer thermal source and a multi-wall thermal conductive layer.

Materials can be classified into opaque or electrical conductors, transparent or low dielectric loss materials, absorbers or high dielectric loss materials. ${ }^{13}$ Two types of pavement (Cement concrete and Asphalt concrete) are used in this work. Generally, the material of pavement and water are regarded as absorbers which assimilate 
MV energy based on the value of the high dielectric loss factor and convert it to heat, while the ice can be approximately regarded as transparent, in which MW are neither reflected nor absorbed, but transmit with little attenuation. ${ }^{13}$ Therefore, the frozen portion of the ice layer absorbs less MW energy than thawed portion in respect to certain MW frequency, the energy absorbed by the pavement is much less than those of water as well. ${ }^{14}$

Generally, the transmission temperature of water between solid state and liquid state is $273 \mathrm{~K}$. Exposed to low temperature below $273 \mathrm{~K}$, what obstructs the ice layer detaching easily from the pavement is the freezing adhesion caused by ice phase change. Freezing adhesion between ice layer and the surface of pavement, developing in the low temperature below 0 , is mainly governed by the surface roughness. ${ }^{15}$ The frozen water crams the cracks in the rough surface, leading to the strong adhesion between the ice layer and road surface. However, the layer can be easily separated from those while frozen water in these cracks thawed out. For most highways and airport runways in China, the roughness of these surfaces is regarded as $1 \mathrm{~mm} .{ }^{16}$ Thus, the evaluation of deicing is obtained that the vertical height of thawed water must reach a quarter of the roughness in the longitudinal surface of ice layer, which is considered as the standard evaluation of deicing.

In this study, a feasible analytic model of MV deicing is established that takes multiple factors into consideration, such as ice thickness, ambient conditions, materials properties of pavement and energy generation. The analytic solutions of MV deicing progress are developed considering the non-heterogeneous heating for the underground, the phase transfer of water and heat convection between the top surface of ice layer and the flowing air. The temperature field caused by transient heat conduction is calculated by quantitative analysis. The specific objectives of this study were to develop a coupled electromagnetic and transient heat transfer model with phase change using finite difference time-domain, and further analyze the factors in deicing efficiency, determine the optimal parameters of this deicing system.

\section{Mathematical Model of Deicing Process}

The melting process involves the coupled equations governing electromagnetic distribution and heat transfer. To solve Maxwell's equations coupled with heat transfer equation, it is necessary to set the temperature-dependent material properties, such as the electrical permittivity, $\varepsilon(T)$; specific heat, $c(T)$ and thermal conductivity, $\rho(T)$. For the sake of simplifying the calculation and achieving a certain accuracy level under some specific conditions, the pavement deicing analysis system is regarded as a one-dimensional analytical model, which means the MW and heat flux transmits vertically to the surface of pavement, and take no account of other directional transmission. As a result, the heating transfer process with MW melting is provided in Fig. 1. The entire heat transfer process could be split into double processes. 


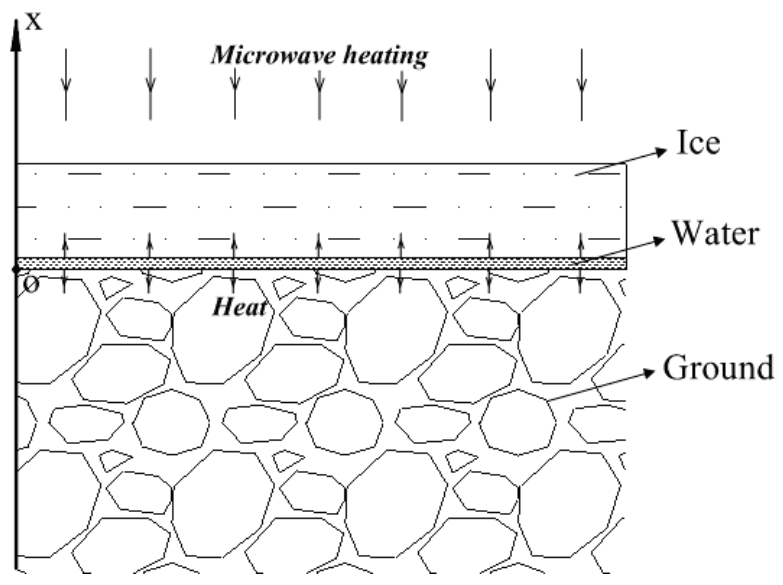

Fig. 1. One-dimensional MW deicing process.

Firstly, the MW irradiates at the icy road. Owing to greater transmittance of the solid ice layer, most energy is absorbed by the road. ${ }^{17}$ Regarding the cement concrete as the first researching material of the pavement, there is no phase change in this process until the temperature of the interface between the pavement and ice layer reaching $273 \mathrm{~K}$. In addition, the bottom of the ice layer begins to thaw while the temperature of the interface is close to $273 \mathrm{~K}$. Because of the high dielectric loss of water, its MW energy absorbing ability which is much stronger than cement cannot be neglected. Meanwhile, the melting rate will increase properly. Moreover, during the heating process, the natural convection is formed between the top surface of ice layer and the flowing air. The temperature of this surface and air is equivalent of initial time. However, with the temperature of the top surface of the ice increasing, heat convection generates and some parts of thermal energy is dissipated into the air, owing to the heat transfer from the lower part, and temperature difference between the ice and the air.

\subsection{Model of electromagnetic energy sources}

The study of the MWs generated by magnetron corresponds to a frequency of $5.8 \mathrm{GHz}$ for deicing. The proposed model is based on the following assumptions:

(i) The absorptions of MW energy by flowing air and solid ice layer are negligible, and the MW transmits with no attenuation.

(ii) The MV propagate terminates in a critical depth under the pavement.

(iii) The resonances caused by the reflected wave and transmission wave at the interfaces is ignored inside the material. ${ }^{18}$

(iv) A one-dimensional model over longitudinal directions is practicable to analyzing the electromagnetic field. ${ }^{19}$ 
Numerical analysis on thermal characteristics and ice melting efficiency

Table 1. Partial parameters of thermal and dielectric properties.

\begin{tabular}{|c|c|c|}
\hline Parameters & Value & Description \\
\hline$f$ & 5.8 & Microwave frequency $(\mathrm{GHz})$ \\
\hline$\varepsilon_{c}$ & 8 (Ref. 26) & $\begin{array}{l}\text { Complex relative permittivity of concrete } \\
\text { cement }\end{array}$ \\
\hline$\varepsilon_{w}^{\prime}$ & $\begin{array}{l}44.6283+1.39298 T-0.032226 T^{2}+3.16517 \\
\quad \times 10^{-4} T^{3}-1.50321 \times 10^{-4} T^{4}(\text { Ref. } 31)\end{array}$ & Complex relative permittivity of water \\
\hline$\varepsilon_{w}^{\prime \prime}$ & $\begin{array}{l}40.5733-0.14756 T-0.02477 T^{2}+6.09212 \\
\quad \times 10^{-4} T^{3}-6.00015 \times 10^{-4} T^{4}(\text { Ref. } 31)\end{array}$ & Complex relative permittivity of water \\
\hline$c_{c}$ & $798-2.1 T$ (Ref. 26$)$ & Specific heat of concrete cement $(\mathrm{J} / \mathrm{kg} \cdot \mathrm{K})$ \\
\hline$c_{i}$ & $2.114+0.007789 T$ (Ref. 31) & Specific heat of ice $(\mathrm{J} / \mathrm{kg} \cdot \mathrm{K})$ \\
\hline$c_{w}$ & $4.109+0.01 T-0.004 T^{2}($ Ref. 32$)$ & Specific heat of water $(\mathrm{J} / \mathrm{kg} \cdot \mathrm{K})$ \\
\hline
\end{tabular}

The wave equation which defined the electromagnetic distribution in space and time can be numerically solved by Maxwell's equations. The differential form of Maxwell's equations can be expressed in terms of the electric field intensity $E$ and the magnetic field density $H .^{20}$

$$
\begin{aligned}
\nabla \times E & =-\frac{\partial(\mu H)}{\partial t}, \\
\nabla \times H & =\omega \varepsilon_{0} \varepsilon^{\prime \prime} E+\frac{\partial(\varepsilon E)}{\partial t}, \\
\nabla \cdot \varepsilon E & =\rho, \\
\nabla \cdot H & =0
\end{aligned}
$$

where $\varepsilon_{0}$ and $\varepsilon^{\prime \prime}$ are the free space permittivity $\left(8.854 \times 10^{-12} \mathrm{~F} / \mathrm{m}\right)$ and the dielectric loss; $\omega$ is the angular frequency; $\mu$ is the permeability.

The dielectric constant $\varepsilon^{\prime}$ reflects the ability of the material to store electric energy, whereas dielectric loss $\varepsilon^{\prime \prime}$ influences the conversion of electromagnetic energy into thermal energy. ${ }^{21}$ The complex relative permittivity, $\varepsilon$, of the dielectric material is given by

$$
\varepsilon=\varepsilon^{\prime}+j \varepsilon^{\prime \prime},
$$

where $\varepsilon^{\prime}$ is the dielectric constant and dielectric loss factor. For these materials, we can expect the magnetic susceptibility to vary with the temperature. ${ }^{22}$ Both $\varepsilon^{\prime}$ and $\varepsilon^{\prime \prime}$ are temperature $(T)$ dependent and a number of investigations have been made in order to define this behavior. ${ }^{23}$ As is shown in Table 1 , the corresponding parameters are shown in Table 1 . In most cases, their values increase as the material thaws and thereafter decrease as the temperature increases.

In addition to the reflected MW on the surface, the electromagnetic power gradually dissipates along the direction of penetration. The heat absorbed by the sample per unit time due to the MWs is given $b^{24}$

$$
Q^{\prime}(x, t)=\frac{1}{2} \omega \varepsilon_{0} \varepsilon^{\prime \prime}|E|^{2} .
$$


Since the volumetric power absorption is proportional to the dielectric loss, and the penetration depth, which can be reduced with increasing the value of dielectric loss, is calculated by ${ }^{25}$

$$
L_{p}=\frac{\lambda_{0}}{2 \pi \sqrt{\varepsilon^{\prime}}}\left[\sqrt{\left\{1+\left(\frac{\varepsilon^{\prime \prime}}{\varepsilon^{\prime}}\right)^{2}\right\}}-1\right]^{-0.5} .
$$

The pavement of this deicing system is considered as a semi-infinite slab. The computational domain in the longitudinal direction which is the critical depth is calculated by ${ }^{26}$

$$
L_{c}=2.7 L_{p}-0.08
$$

\subsection{Model of heat transfer}

In order to reduce the complexity of analyzing the process of heat transport due to the MW heating, several assumptions have been introduced into the heat equations:

(i) Assuming the ice is immobile, without rise and decline during deicing process, and the whole process is symmetrical.

(ii) Heat conduction process under the critical depth has no influence on heat transfer of deicing process.

(iii) Ignoring the influence of the natural-convection on the ice layer's shape and the influence of the vehicle speed on the air convection heat transfer coefficient.

(iv) Ignoring the convection between ice and water when the phase of ice has changed.

(v) Ignoring the heat loss to the ambient road. The heat exchanging of pavement is due to the surface connection to the ice layer.

While the temperature difference is generated between different objects, the thermal energy would transfer from the high-temperature object to the lowtemperature object or from the high-temperature section to the low-temperature section. Different genres of heat transfer process are called different heat transfer patterns. Temperature distributions in this object which is exposed to the MW are predicted by the energy balance equation. ${ }^{27}$

$$
\dot{E}_{\text {st }}=\dot{E}_{\text {in }}-\dot{E}_{\text {out }}+\dot{E}_{g}
$$

where $E_{\text {st }}$ represents the stored thermal energy and mechanical energy; $E_{\text {in }}$ represents the entering energy from adjacent unit; $E_{\text {out }}$ represents the lost energy leaving for the adjacent unit, while $E_{g}$ is the produced thermal energy from other factors.

Based on heat transport due to conduction, the governing partial differential equation for the temperature in the sample as a function of space and time obtained by this energy conservation included convection, diffusion, conduction, phase change of water, and MW heating source is given by ${ }^{28}$

$$
\rho(x, T) c(x, T) \frac{\partial T(x, t)}{\partial t}=k(x, T) \frac{\partial^{2} T(x, t)}{\partial^{2} x}+Q^{\prime}(x, t), \quad 0 \leq x \leq L_{i},
$$




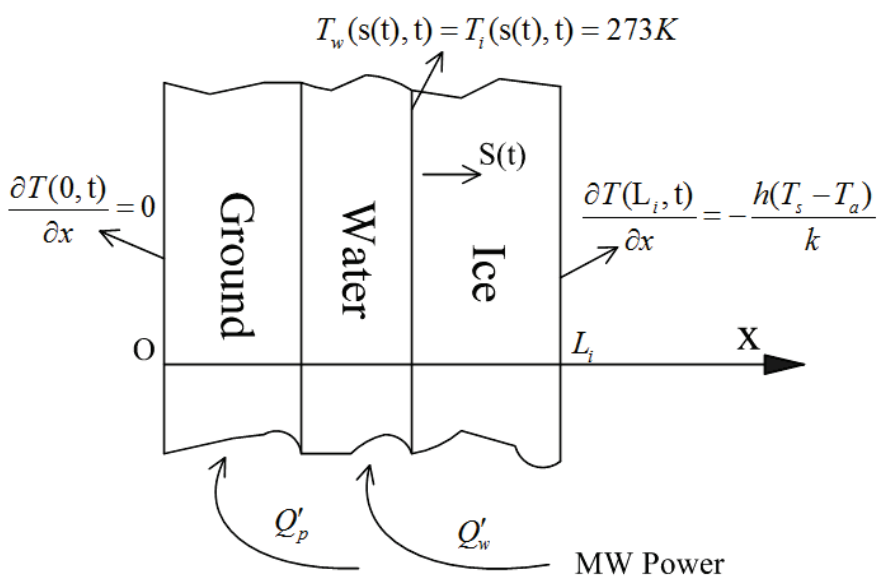

Fig. 2. The schematic of the deicing process with ice phase change.

where $k(x, T)$ is the thermal conductivity, $c(x, T)$ is the function of specific heat and $\rho(x, T)$ is the material density.

When the temperature at the interface between the pavement and the ice layer increases to $273 \mathrm{~K}$, the bottom part of this layer ice begins to change the phase and melts gradually. As Fig. 2 shows, the $\rho(x, T)$ can be expressed as follows, and $c(x, T), k(x, T), \varepsilon(x, T), \varepsilon^{\prime \prime}(x, T)$ meet these rules as well. The relevant parameters of different materials are represented in Table 1.

$$
\rho(x, T)= \begin{cases}\rho_{p}(x, T) ; & 0<x \leq L_{c}, \\ \rho_{i}(x, T) ; & L_{c}<x \leq L_{i}, \quad T<0, \\ \rho_{w}(x, T) ; & L_{c}<x \leq L_{i}, \quad T>0 .\end{cases}
$$

In the equation, $\rho_{p}(x, T), \rho_{i}(x, T)$ and $\rho_{w}(x, T)$ respectively represent the density of pavement material, ice layer and water of which the temperature exceeds $273 \mathrm{~K}$.

\subsection{Initial and boundary conditions}

In the one-dimensional plane, wind at the ice surface enhances heat transfer and carries away heat with the fluid flow, which is characterized by a convection coefficient in the theory of convection heat transfer as a kind of boundary condition. And the part below critical depth under pavement is supposed to be insulated. Therefore, the general boundary condition on the material's surface can be expressed as

$$
\begin{aligned}
& -k(T) \frac{\partial T}{\partial x}=h\left(T_{s}-T_{a}\right), \quad x=L_{i}, \\
& -k(T) \frac{\partial T}{\partial x}=0, \quad x=L_{c},
\end{aligned}
$$


where $T_{s}$ is the temperature of the top surface of ice layer, $T_{a}$ is the convective air temperature and $\hbar$ is the convective heat transfer coefficient.

The wind speed on deicing is the key effect for the convection coefficient, which can be expressed with the following power equation. ${ }^{29}$

$$
h=\frac{\lambda}{l} \mathrm{Nu}=\frac{\lambda}{l}\left[C \cdot \operatorname{Pr}^{n} \cdot \mathrm{Gr}^{m} \cdot \operatorname{Re}^{k}\right],
$$

where $\mathrm{Nu}$ is the Nusselt number, which depends on the intensity of the convection heat transfer between the top surface of ice layer and the flowing air; $\operatorname{Pr}$ is the Prandtl number, which indicates the properties of the fluid. Re is the Reynolds number, which is used to indicate the fluid states, and $\mathrm{Gr}$ is Grashof number, which is used to determine the effect of natural convection on heat transfer.

The convection coefficient is effected by many parameters, such as wind speed, air density, air viscosity and ambient temperature. ${ }^{30}$ When the wind speed is far lower than $20 \mathrm{~m} / \mathrm{s}$, the Reynolds number meets $\mathrm{Re}<1.38 e 5$. The Nusselt number should be calculated by the format as follows. ${ }^{9}$

$$
\mathrm{Nu}=\frac{L h}{\lambda}=0.664 \operatorname{Re}^{1 / 2} \operatorname{Pr}^{1 / 3} .
$$

Therefore, the convection coefficient can be simplified: ${ }^{9}$

$$
h=3.78\left(\frac{v}{l}\right)^{1 / 2},
$$

where $v$ is the wind speed and $l$ is the length of the wind flow.

The initial conditions for this model considered here are as follows and is coincident with the ambient temperature based on neglecting the slight temperature gradient in the deicing systems.

$$
\begin{aligned}
& T_{p}(t=0)=T_{e}, \quad 0 \leq x \leq L_{c}, \\
& T_{i}(t=0)=T_{e}, \quad L_{c}<x \leq L_{i},
\end{aligned}
$$

where $T_{e}$ is the ambient temperature.

\section{Model Solving}

\subsection{Finite differential method}

Numerical analysis of MW deicing process was applied by finite element code using finite difference method. The partial differential equation of each node could be obtained through the energy conservation, and the temperature characteristics of each nodes could be estimated rapidly by using the numerical method, which could help to analyze the variation of the temperature field under different conditions and obtain the precise result of the sophisticated one-dimensional geometrical shape which involves different types of boundary conditions. As a result, the energy conservation finite difference equation of each node could be obtained through the 
finite differential method, thus the temperature distribution of each node could be calculated.

When calculating the critical depth, set the $5 \mathrm{~mm}$-depth cement concrete as the study object, and divide the ice layer into $m$ nodes. Then separate the ice layer of $5 \mathrm{~cm}$ thickness into $n$ nodes and make dissociation of both the position and the time. Then introduce integer $p$.

$$
\begin{aligned}
t & =p \Delta t, \\
\left.\frac{\partial T}{\partial t}\right|_{m(n)} & \approx \frac{T_{m(n)}^{p+1}-T_{m(n)}^{p}}{\Delta t}, \\
\left.\frac{\partial^{2} T}{\partial x^{2}}\right|_{m(n)} & \approx \frac{\partial T /\left.\partial x\right|_{m+1(n+1)}-\partial T /\left.\partial x\right|_{m-1(n-1)}}{\Delta x} \\
& =\frac{T_{m+1(n+1)}+T_{m-1(n-1)}-T_{m(n)}}{(\Delta x)^{2}} .
\end{aligned}
$$

When $x=L_{c}$, road surface is set as the 0 node. The ice layer on top of the road surface is 1 to $m$ node. Whereas, the $5 \mathrm{~cm}$ range of critical depth under the pavement is set as $m$ to $n$ node $(m<n)$. The finite difference equation at the 0 node could be obtained by using the energy conservation equation:

$$
\frac{T_{0}^{p+1}-T_{0}^{p}}{\Delta t}=\frac{k_{c}}{\rho_{c} c_{c}} \frac{T_{1}^{p+1}-T_{0}^{p+1}}{(\Delta x)^{2}}+\frac{k_{i}}{\rho_{c} c_{c}} \frac{T_{m+1}^{p+1}-T_{0}^{p+1}}{(\Delta x)^{2}}+\frac{Q_{c}^{\prime}(0, t)}{\rho_{c} c_{c}} .
$$

When $x>L_{c}$, the plane stays on the ice layer section, the ice layer absorbs thermal energy from the cement concrete before the phase changes. The finite difference equation of each node in the ice layer could be obtained through the energy conservation equation as well.

$$
\begin{aligned}
\frac{T_{1}^{p+1}-T_{1}^{p}}{\Delta t} & =\frac{k_{i}}{\rho_{i} c_{i}} \frac{T_{2}^{p+1}+T_{0}^{p+1}-2 T_{1}^{p+1}}{(\Delta x)^{2}} \\
\frac{T_{2}^{p+1}-T_{2}^{p}}{\Delta t} & =\frac{k_{i}}{\rho_{i} c_{i}} \frac{T_{3}^{p+1}+T_{1}^{p+1}-2 T_{2}^{p+1}}{(\Delta x)^{2}} \\
\frac{T_{3}^{p+1}-T_{3}^{p}}{\Delta t} & =\frac{k_{i}}{\rho_{i} c_{i}} \frac{T_{4}^{p+1}+T_{2}^{p+1}-2 T_{3}^{p+1}}{(\Delta x)^{2}} \\
\frac{T_{m-1}^{p+1}-T_{m-1}^{p}}{\Delta t} & =\frac{k_{i}}{\rho_{i} c_{i}} \frac{T_{m}^{p+1}+T_{m-2}^{p+1}-2 T_{m-1}^{p+1}}{(\Delta x)^{2}} .
\end{aligned}
$$

In this process, as the temperature of the interface between pavement and ice layer rise to $273 \mathrm{~K}$, the bottom part of the ice layer begins to melt. The energy absorbing ability of water is better than the cement concrete by comparing the dielectric properties between them. 
As a result, the pavement material and the water would absorb MW energy at the same time after the ice melts into water. As a certain amount of ice above the interface melts into water, the ice layer will not adhere to the road. Then the MW heating is used to separate the ice from the pavement. The MW act on not only cement concrete but also water, thus, the deicing efficiency is enhanced dramatically. Therefore, considering the water's MW absorbing ability, the MW energy $Q_{w}^{\prime}(x, t)$ absorbed by the water is added to deicing system of difference equation of each node in the water layer.

Assuming node 1 fully melts into water at $t_{0}$, the energy conservation equation is

$$
\frac{T_{1}^{p+1}-T_{1}^{p}}{\Delta t}=\frac{k_{i}}{\rho_{i} c_{i}} \frac{T_{2}^{p+1}+T_{0}^{p+1}-2 T_{1}^{p+1}}{(\Delta x)^{2}}+\frac{Q_{w}^{\prime}\left(1, t_{1}\right)}{\rho_{i} c_{i}} .
$$

The temperature of each node at $t_{1}$ is set as the initial temperature, then calculate the finite difference equation when the cement concrete and water absorb MW energy at the same time. Analyze the node 2 through this method, and at the moment $t_{2}$ when the node 2 fully melts into water, the characteristic of the node 1 after absorbing the MW energy is initial temperature of the energy conservation equation $Q_{w}^{\prime}(2, t)$. The energy conservation equation at the node 2 is obtained:

$$
\frac{T_{2}^{p+1}-T_{2}^{p}}{\Delta t}=\frac{k_{i}}{\rho_{i} c_{i}} \frac{T_{3}^{p+1}+T_{1}^{p+1}-2 T_{2}^{p+1}}{(\Delta x)^{2}}+\frac{Q_{w}^{\prime}\left(2, t_{2}\right)}{\rho_{i} c_{i}} .
$$

When $x=L_{i}$, the position is the top surface of the ice layer, the thermal energy transfers in the positive direction of $x$ to the top surface of the ice layer node $m$. Considering the energy change at the node $m$ at the surface of the ice layer, the natural-convection is formed at the surface of the ice layer by the air flow. The finite difference equation at node $m$ could be obtained through the energy conservation equation:

$$
\frac{T_{m}^{p+1}-T_{m}^{p}}{\Delta t}=\frac{k_{i}}{\rho_{i} c_{i}} \frac{T_{m-1}^{p+1}-T_{m}^{p+1}}{(\Delta x)^{2}}+\frac{h}{\rho_{i} c_{i}} \frac{\left(T_{e}-T_{m}^{p+1}\right)}{\Delta x} .
$$

When $x<L_{c}$, the plane stands on the cement concrete section, the temperature rises when the thermal energy absorbed by the cement concrete during MW heating. The MW energy is absorbed with decay along the $x$-axis downward. The finite difference equation system of each node under the depth of $15 \mathrm{~cm}$ could be obtained through the energy conservation equation:

$$
\begin{aligned}
& \frac{T_{m+1}^{p+1}-T_{m+1}^{p}}{\Delta t}=\frac{k_{c}}{\rho_{c} c_{c}} \frac{T_{0}^{p+1}+T_{m+2}^{p+1}-2 T_{m+1}^{p+1}}{(\Delta x)^{2}}+\frac{Q_{c}^{\prime}(m+1, t)}{\rho_{c} c_{c}} \\
& \frac{T_{m+2}^{p+1}-T_{m+2}^{p}}{\Delta t}=\frac{k_{c}}{\rho_{c} c_{c}} \frac{T_{m+3}^{p+1}+T_{m+1}^{p+1}-2 T_{m+2}^{p+1}}{(\Delta x)^{2}}+\frac{Q_{c}^{\prime}(m+2, t)}{\rho_{c} c_{c}}
\end{aligned}
$$




$$
\begin{gathered}
\frac{T_{m+3}^{p+1}-T_{m+3}^{p}}{\Delta t}=\frac{k_{c}}{\rho_{c} c_{c}} \frac{T_{m+4}^{p+1}+T_{m+2}^{p+1}-2 T_{m+3}^{p+1}}{(\Delta x)^{2}}+\frac{Q_{c}^{\prime}(m+3, t)}{\rho_{c} c_{c}} \\
\vdots \\
\frac{T_{m+n-1}^{p+1}-T_{m+n-1}^{p}}{\Delta t}=\frac{k_{c}}{\rho_{c} c_{c}} \frac{T_{m+n}^{p+1}+T_{m+n-2}^{p+1}-2 T_{m+n-1}^{p+1}}{(\Delta x)^{2}}+\frac{Q_{c}^{\prime}(m+n-1, t)}{\rho_{c} c_{c}} .
\end{gathered}
$$

When phase of ice layer starts to change, and the conditions of $L_{c}<x<L_{i}$ and $T>273 \mathrm{~K}$ are satisfied, which describes the section of water between ice layer and pavement, the energy absorbed by water must be considered, which accelerates the speed of ice melting. The energy conservation equation referring to the section of water is expressed by

$$
\begin{aligned}
\frac{T_{m+1}^{p+1}-T_{m+1}^{p}}{\Delta t} & =\frac{k_{w}}{\rho_{w} c_{w}} \frac{T_{0}^{p+1}+T_{m+2}^{p+1}-2 T_{m+1}^{p+1}}{(\Delta x)^{2}}+\frac{Q_{w}^{\prime}(m+1, t)}{\rho_{w} c_{w}} \\
\frac{T_{m+2}^{p+1}-T_{m+2}^{p}}{\Delta t} & =\frac{k_{w}}{\rho_{w} c_{w}} \frac{T_{m+3}^{p+1}+T_{m+1}^{p+1}-2 T_{m+2}^{p+1}}{(\Delta x)^{2}}+\frac{Q_{w}^{\prime}(m+2, t)}{\rho_{w} c_{w}} \\
\frac{T_{m+3}^{p+1}-T_{m+3}^{p}}{\Delta t} & =\frac{k_{w}}{\rho_{w} c_{w}} \frac{T_{m+4}^{p+1}+T_{m+2}^{p+1}-2 T_{m+3}^{p+1}}{(\Delta x)^{2}}+\frac{Q_{w}^{\prime}(m+3, t)}{\rho_{w} c_{w}} \\
\frac{T_{m+n-1}^{p+1}-T_{m+n-1}^{p}}{\Delta t} & =\frac{k_{w}}{\rho_{w} c_{w}} \frac{T_{m+n}^{p+1}+T_{m+n-2}^{p+1}-2 T_{m+n-1}^{p+1}}{(\Delta x)^{2}}+\frac{Q_{w}^{\prime}(m+n-1, t)}{\rho_{w} c_{w}}
\end{aligned}
$$

In this research, matrix inversion method of the direct method is used to solve the finite difference equation. The matrix inversion method is an equation system which has $N$ pieces of finite difference equation response to $N$ pieces of unknown temperature. Single integer subscript is used in the equation to represent the node rather than using double subscript of $(m, n)$. The first step of matrix inversion process is listing these equations:

$$
\begin{gathered}
a_{11} T_{1}+a_{12} T_{2}+a_{13} T_{3}+\cdots+a_{1 N} T_{N}=C_{1}, \\
a_{21} T_{1}+a_{22} T_{2}+a_{23} T_{3}+\cdots+a_{2 N} T_{N}=C_{2}, \\
\vdots \\
a_{N 1} T_{1}+a_{N 2} T_{2}+a_{N 3} T_{3}+\cdots+a_{N N} T_{N}=C_{N} .
\end{gathered}
$$

In the equation, $a_{11}, a_{12}, \ldots, C_{1}, \ldots$ is the known coefficients and constants applying to $\Delta x, k, h$ and $T_{\infty}$. The equation could be represented, using matrix notion:

$$
[A][T]=[C] .
$$


According to this equation, the details can be represented as follows.

$$
A=\left[\begin{array}{cccc}
a_{11} & a_{12} & \cdots & a_{1 N} \\
a_{21} & a_{22} & \cdots & a_{2 N} \\
\vdots & \vdots & & \vdots \\
a_{N 1} & a_{N 2} & \cdots & a_{N N}
\end{array}\right], \quad T=\left[\begin{array}{c}
T_{1} \\
T_{2} \\
\vdots \\
T_{N}
\end{array}\right], \quad C=\left[\begin{array}{c}
C_{1} \\
C_{2} \\
\vdots \\
C_{N}
\end{array}\right] .
$$

The coefficient matrix $[A]$ is a square matrix $(N \times N)$, its elements are represented by double subscript, the first subscript represents row, and the second subscript represents line.

The (unknown) solution's vector could be represented as

$$
[T]=[A]^{-1}[C] \text {. }
$$

In the equation, $[A]^{-1}$ is the inverse matrix of $[A]$ and is defined as

$$
[A]^{-1}=\left[\begin{array}{cccc}
b_{11} & b_{12} & \cdots & b_{1 N} \\
b_{21} & b_{22} & \cdots & b_{2 N} \\
\vdots & \vdots & & \vdots \\
b_{N 1} & b_{N 2} & \cdots & b_{N N}
\end{array}\right]
$$

Calculate to the right side of Eq. (21), the result is as follows:

$$
\begin{gathered}
T_{1}=b_{11} C_{1}+b_{12} C_{2}+\cdots+b_{1 N} C_{N} \\
T_{2}=b_{21} C_{1}+b_{22} C_{2}+\cdots+b_{2 N} C_{N} \\
\vdots \\
T_{N}=b_{N 1} C_{1}+b_{N 2} C_{2}+\cdots+b_{N N} C_{N} .
\end{gathered}
$$

After calculating the inversion of $[A]$, the elements of $b_{11}, b_{12}, \ldots$ could be obtained, and all of the unknown parameters of temperature are calculated from the upper equation.

\subsection{Solution procedure}

Transient heat transfer with MW heating is synchronously simulated on the base of $1 \mathrm{D}$ environment, while material thermal and dielectric properties with phase change are updated at the time $t$. The physics coupling and the solution process are illustrated in Fig. 3 and are now discussed in detail.

The material properties used to solve heat transfer were updated as temperature value functions. The longitudinal temperature distribution of ice layer and ground in critical depth can be calculated without considering phase change, with ground being the only object absorbing MW energy. The calculation will maintain this way until phase begins changing, when water and ground, which could both absorb MW power, are both considered as energy source of this model. This process will keep operating until the melted part of ice layer meets the deicing requirement mentioned above. Finally, the solutions at each moment are obtained, and the temperature distribution under all conditions will show the influence factors on deicing efficiency. 


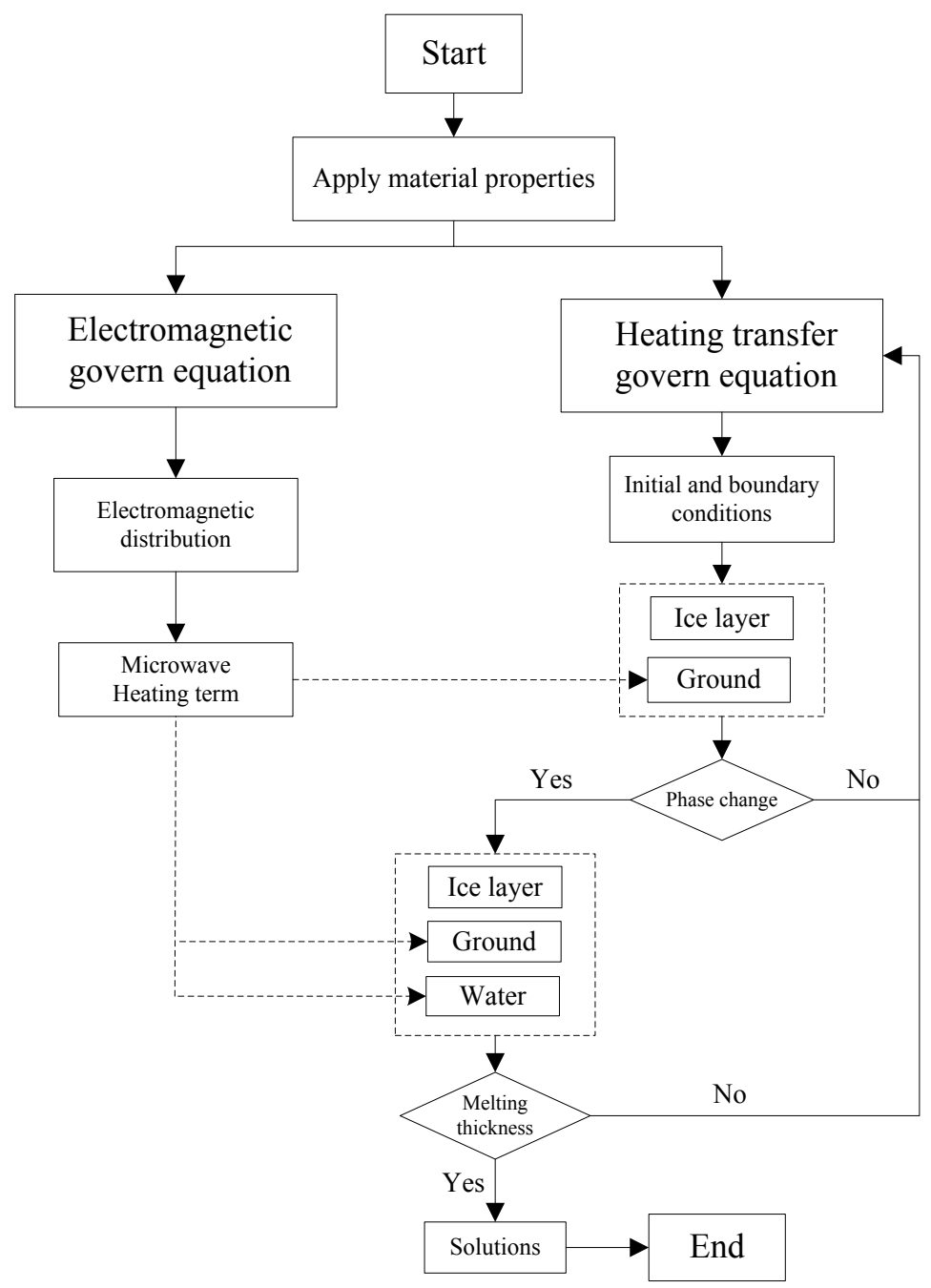

Fig. 3. A flowchart of solution procedure.

\section{Numerical Analysis}

According to the established deicing heat transfer model, the ice layer's variation and the distribution of the temperature field during the MW deicing process are presented. Then the influence factors among the ice layer's thickness, ambient temperature, absorbed power and deicing time are analyzed. In this way, velocity of deicing vehicle, bottom plate's length, MW power, ambient temperature and ice layer's thickness could be matched under an appropriate condition.

Analyze the deicing heat transfer model with the finite difference method to obtain the distribution of the one-dimensional temperature field in the vertical 


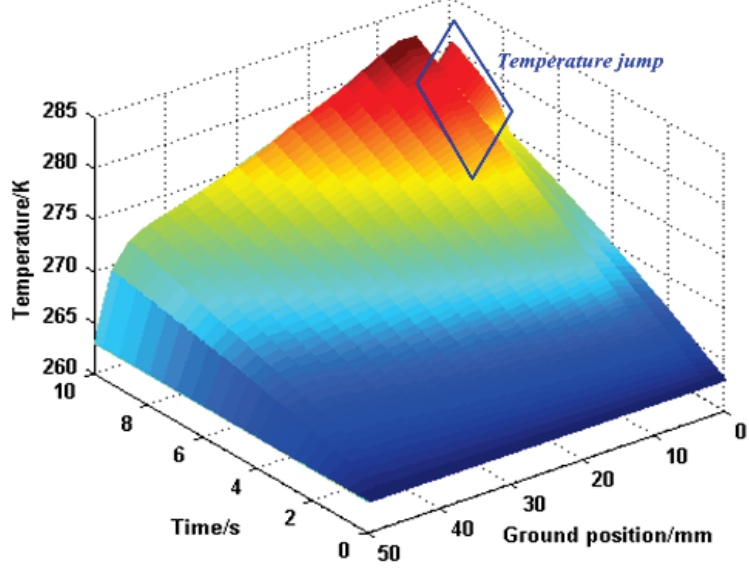

(a) Temperature distribution of $50 \mathrm{~mm}$ ground

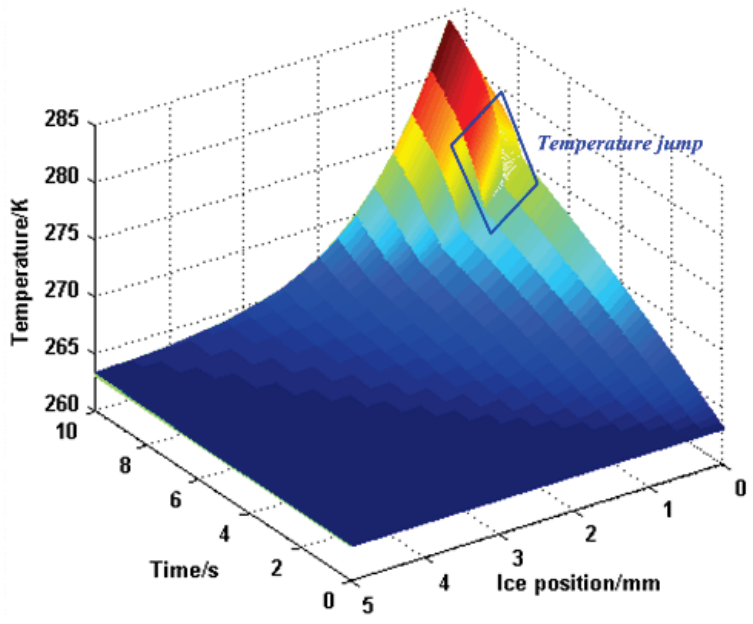

(b) Temperature distribution of $5 \mathrm{~mm}$ ice

Fig. 4. (Color online) Longitudinal temperature distribution of the ice and ground.

direction. Assume the ice layer's thickness $d=5 \mathrm{~mm}$, the environment temperature is $263 \mathrm{~K}$. When the ice layer's step size is $\Delta x=0.00025 \mathrm{~mm}, m=20$, set the time interval $\Delta t=0.01 \mathrm{~s}$; then set the step size under the depth of $50 \mathrm{~mm}$ of $\Delta x=0.0025 \mathrm{~mm}, n=20$, set the time interval $\Delta t=0.01 \mathrm{~s}$. After bringing the absorbed power into the model, the temperature variation of each node can be obtained. Then establish the distribution of temperature field in the vertical length during MW heating deicing process, which is shown in Fig. 4. According to Fig. 4(a), with the decrease of the depth, the temperature raising rate of each node in the positive direction of $x$-axis increases, and the temperature of each node under road increases with enhancement in this heating process. The zenith of the temperature 
is under the depth of $0-5 \mathrm{~mm}$, and the majority of MW energy is absorbed by the road through the ice layer. The temperature of the cement concrete is lower below the depth of $20 \mathrm{~mm}$, which means that fewer MW energy is absorbed by this part, which will help reduce the MW's energy loss in the cement concrete and enhance the efficiency of ice layer's melting process at the juncture of the road.

According to Fig. 4(b), before $8 \mathrm{~s}$, the temperature raising law of the nodes in the ice layer is the same as those under the road. After $8 \mathrm{~s}$, the ice layer at the depth of $1 \mathrm{~mm}$ will have a temperature jump, which results from parts of the ice experiencing phase change and melting into water after $8 \mathrm{~s}$. Moreover, water dielectric constant and dielectric loss angle are large. According to Eq. (3), water absorbs more MW energy than the cement concrete in the same electric field, and water transfers more thermal energy, which intrigues temperature jump in the ice layer and increases the temperature raising rate dramatically. Finally, in the figure, after heating about $10 \mathrm{~s}$, the temperature of the ice layer's top surface rises from $1 \mathrm{~K}$ to $264 \mathrm{~K}$. In conclusion, with the environment temperature of $263 \mathrm{~K}$ and the ice layer's thickness of $5 \mathrm{~mm}$, the ice below the depth of $0.25 \mathrm{~mm}$ experiences phase change and the deicing efficient enhances dramatically after $8 \mathrm{~s}$ of heating, while the ice melts about $25 \%$ after 10 .

In order to analyze the relationship between the variation of ice temperature and the temperature distribution below the depth of $50 \mathrm{~mm}$, the property curve of temperature is obtained in Fig. 5, while Fig. 5(a) shows the temperature variation changing with the heating time and Fig. 5(b) presents the longitudinal temperature distribution of the ice layer. According to Figs. 5(a) and 5(b): the temperature of the nodes in the ice layer rises a nonlinear manner which is similar to that in the exponential distribution. At first, the temperature increases at a low speed, and the cure is smooth, which means that the ice absorbs less MW energy while the road temperature is low, also the temperature is not high enough to melt the ice. In the later process, the temperature increase at a high speed, which is because that the road absorbs large amount of MW energy and transfers them into thermal energy, then transmits them to the ice layer. At $7.88 \mathrm{~s}$, the road temperature rises to $273 \mathrm{~K}$, and phase change happens in the ice layer, because water's ability of absorbing MW energy is better than that of the cement concrete. Therefore, the ice layer's temperature jumps at this moment, and the closer to the water layer, the more conspicuous the mutation is. According to the figure, the temperature at the juncture of the pavement and the ice layer decreases, and reaches the minimum at the surface of the ice. Finally, according to Fig. 5(a), the temperature of the surface of the ice is not high enough to melt the ice, which can verify the feasibility of deicing vehicle.

Figure 5(c) shows the temperature variation of these nodes at the vertical plane below the road at various heating times, and Fig. 5(d) presents the longitudinal temperature distribution of the nodes under the road at every moment. According to the figure, for a certain node, the temperature increases mildly during the entire heating phase. Initially, the temperature at the depth of $5 \mathrm{~mm}$ is roughly equivalent 
C. Wang et al.

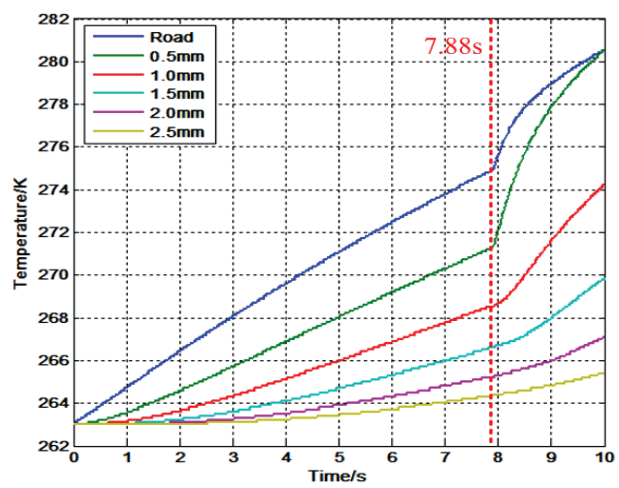

(a) Ice layer's temperature characteristic of each node

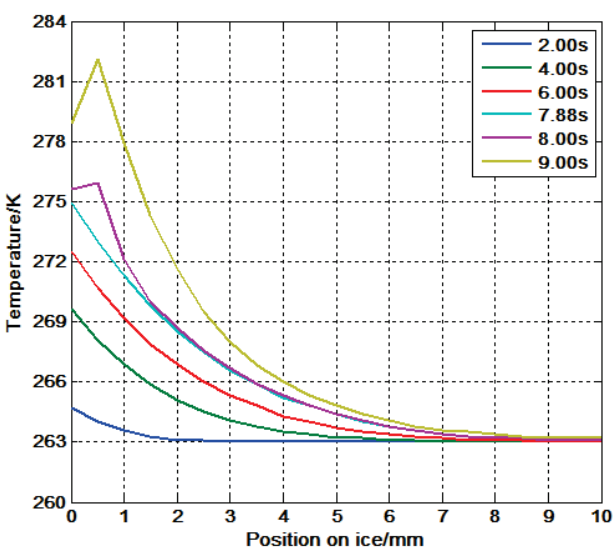

(b) Ice layer's temperature characteristic of each time point

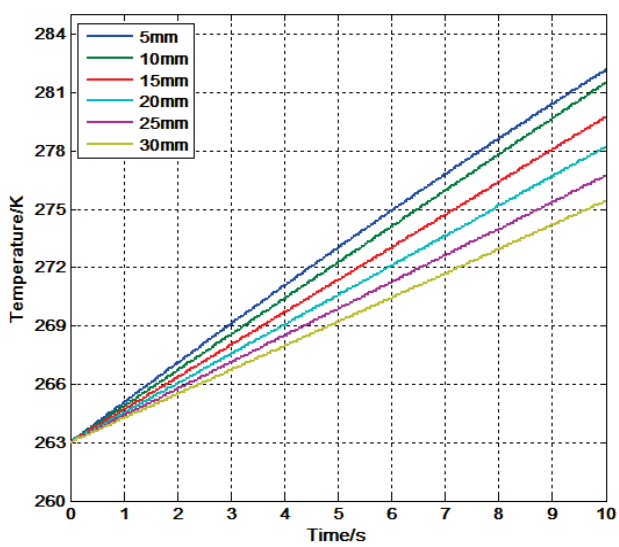

(c) Pavement's temperature characteristic of each node

Fig. 5. (Color online) Characteristics of temperature distributions. 


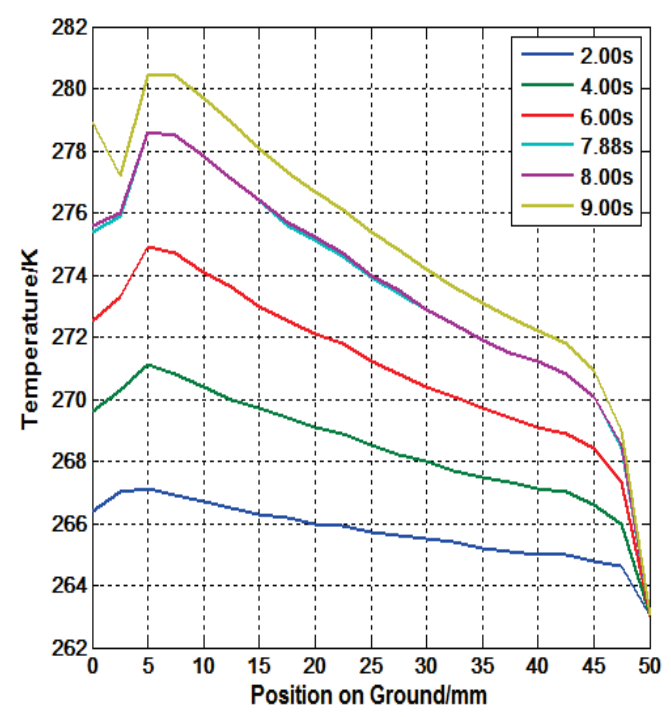

(d) Pavement's temperature characteristic of each time point

Fig. 5 (Continued)

to ambient temperature, which is because the cement concrete at the surface of pavement and the inner part absorbs low rate of energy at first, transferring only little thermal energy to the outer area. To sum up, the ice layer experiences phase change at $7.88 \mathrm{~s}$, and the temperature of pavement frustrates in a small range. With the heating time increases, the bigger part of ice melts, the more energy is absorbed by its liquid part, and the temperature gap between the pavement surface and critical depth widens.

\section{Influencing Parameters Analysis}

In order to analyze the influence of the ice layer's thickness, environment temperature and the power density of the MW on the deicing efficiency, set the moment when the ice layer at the depth of $0.25 \mathrm{~mm}$ changes its phase as the evaluation indicator of the deicing power. Analyze different conditions, when the ice layer's thickness is $5 \mathrm{~mm}, 10 \mathrm{~mm}, 20 \mathrm{~mm}$, the environment temperature is relatively $253 \mathrm{~K}$, $263 \mathrm{~K}, 268 \mathrm{~K}$, and the power density of the MW absorbed by the pavement material is $1.5 \mathrm{MW} / \mathrm{m}^{3}, 2.5 \mathrm{MW} / \mathrm{m}^{3}, 3.5 \mathrm{MW} / \mathrm{m}^{3}$. Calculate the $\mathrm{MW}$ heat transfer model and the temperature field's variation of each node. Then the time needed to melt this ice under different working conditions will be obtained. At last, analyze the image of different conditions.

\subsection{Thickness of ice layer}

Considering the influence of the ice layer thickness to the deicing power, set the power density of the microwave at $3.5 \mathrm{MW} / \mathrm{m}^{3}$, then analyze the variation of the 


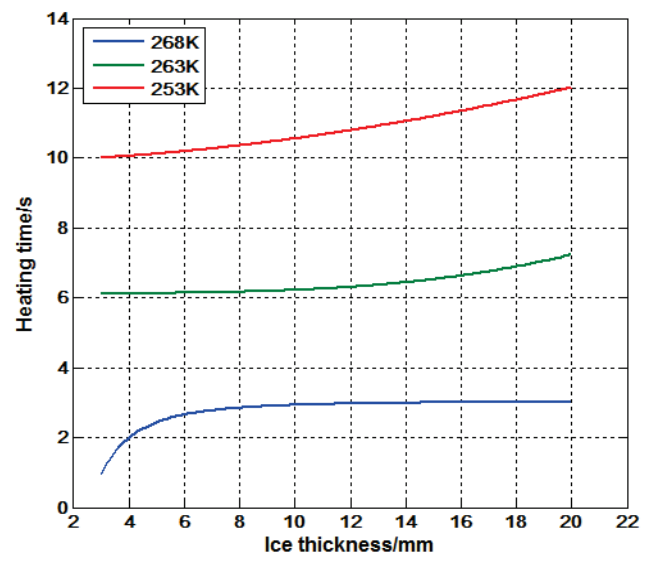

(a) The ice layer's thickness influence when the power density is $3.5 \mathrm{MW} / \mathrm{m}^{3}$

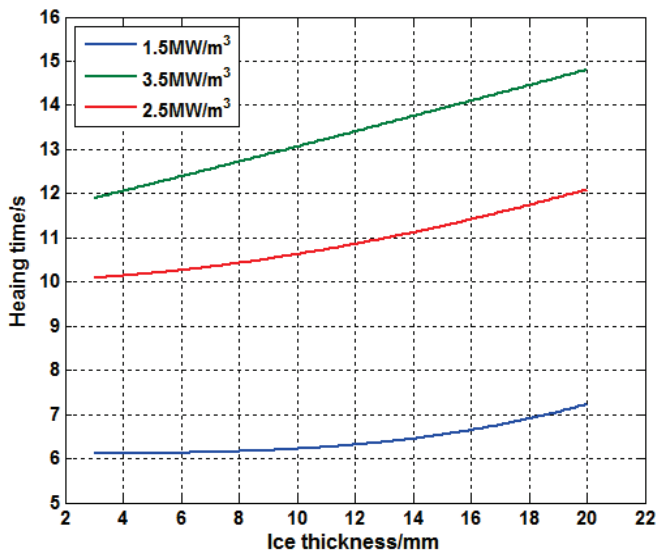

(b) The ice layer's thickness influence when the ambient temperature is $263 \mathrm{~K}$

Fig. 6. (Color online) The influence of the ice layer's thickness.

demand heating time to separate ice from the pavement, which changes with the ambient temperature, while the ice layer's thickness is set to $5 \mathrm{~mm}, 10 \mathrm{~mm}, 20 \mathrm{~mm}$, as is shown in Fig. 6(a). According to the figure, when the environment temperature is the same as the power density, the thicker the ice is, the more time it needs to melt $0.25 \mathrm{~mm}$ of ice. With enhancement of the ice layer's thickness, the rising rate is smooth at first and increases after that. With different environment temperatures, the rising rate of heating time varies with the enhancement of ice, and increases with the enhancement of the environment temperature. When the environment temperature is high, of about $268 \mathrm{~K}$, the thickness of ice layer has a huge impact on the heating time, with the heating time changing inconspicuous when the thickness enhances. The MW's heating time is within $14 \mathrm{~s}$, when the ice layer's thickness ranges from $0 \mathrm{~mm}$ to $20 \mathrm{~mm}$, and the deicing efficiency is relatively high. 
When the environment temperature is $263 \mathrm{~K}$, the MW's power density is set at $1.5 \mathrm{MW} / \mathrm{m}^{3}, 2.5 \mathrm{MW} / \mathrm{m}^{3}, 3.5 \mathrm{MW} / \mathrm{m}^{3}$ relatively, as is shown in Fig. 6(b). With the ice layer's thickness increasing, the MW's heating time grows. According to the figure, when the MW's power density increases, the rising rate of heating time grows as the ice layer's thickness enhances. When the power is at $1.5 \mathrm{MW} / \mathrm{m}^{3}$, the heating time's rising rate is smooth at first and then increases, while the heating time keeps rising when the power is at $3.5 \mathrm{MW} / \mathrm{m}^{3}$. In addition, as it is shown in Fig. 6(b), with the given ambient temperature and the ice thickness being small, the growth rate of heating time increases obviously as the power density decreases. With a fixed ice thickness, the deicing efficiency enhances when the power density increases.

\subsection{Ambient temperature}

With the influence of the environment temperature on deicing efficient considered, analyze it with the MW's heating time when the MW's power density is 3.5 MW/ $\mathrm{m}^{3}$. According to Fig. 7(a), when the ice layer thickness is fixed, the heating time reduces dramatically with the enhancement of environment temperature, also the reduction rate is smooth at first and then increases. The higher the environment temperature is, close to the ice's melting point $273 \mathrm{~K}$, the closer the demand heating time of $5 \mathrm{~mm}$ ice and $10 \mathrm{~mm}$ ice is. When the environment temperature is relatively low, the deicing time increases with ice thickness. In comparison, when the environment temperature is high, the influence of ice layer's thickness on the demand heating time to melt the ice with thickness of $0.25 \mathrm{~mm}$ is little, so is it on the deicing efficiency.

When the ice layer's thickness is $5 \mathrm{~mm}$, as the MW's power density is fixed, the heating time reduces with the enhancement of the environment temperature, according to Fig. 7(b), and the reduction rate is smooth at first then increases. When the environment temperature is high, the demanding heating time is constant, with the MW's power density being $1.5 \mathrm{MW} / \mathrm{m}^{3}, 2.5 \mathrm{MW} / \mathrm{m}^{3}$. When the environment temperature is low, the gap of the demand heating time is wide as the power density changes. To conclude, when the environment temperature is high, the influence of the MW's density on the demand heating time is little, and is it on the deicing efficiency. The lower the environment temperature is, the bigger the influence of MW's density on the deicing is.

\subsection{Pavement material}

Since the MW power absorbing ability is different with diverse materials, the cement concrete and asphalt concrete are comparatively analyzed. The different pavement materials' impact on ice melting efficiency is discussed. As is shown in Fig. 8(a), the heating time decreases gently while the ambient temperature rises. At the same time, the difference between the heating time of cement concrete and asphalt 


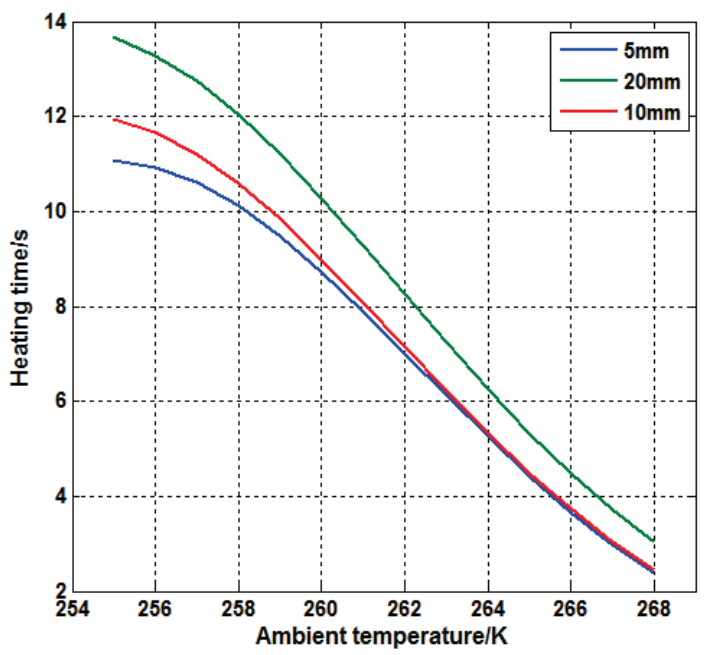

(a) The ambient temperature influence when the power density is $3.5 \mathrm{MW} / \mathrm{m}^{3}$

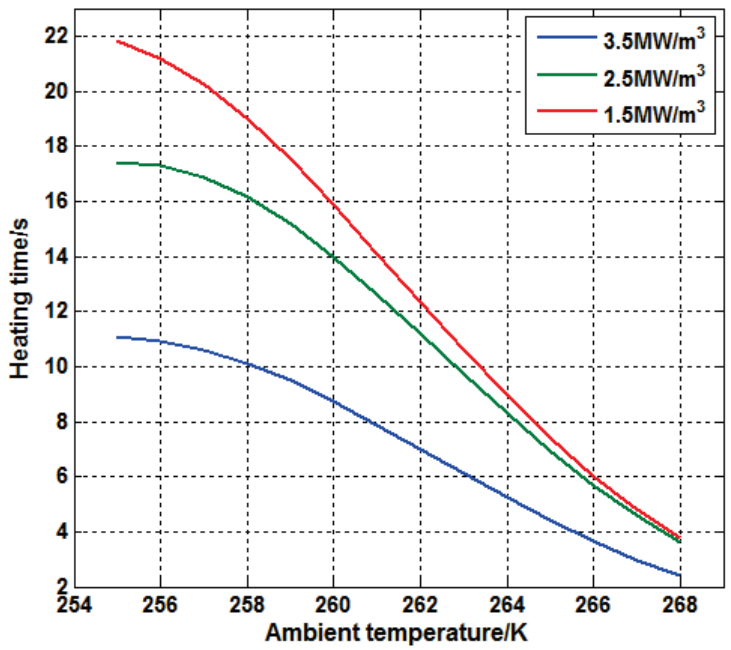

(b) The ambient temperature influence when the ice thickness is $5 \mathrm{~mm}$

Fig. 7. (Color online) The influence of ambient temperature.

concrete increases with the rise of ambient temperature. However, the heating time of asphalt concrete is always about three times as long as that of cement concrete.

Figure 8(b) shows that, with the ice thickness increasing, the MW heating time gradually increases, and the changing rate of heating time of thinner ice increases quietly. With the ice thickness increasing, the changing rate increases significantly, so does the heating time, while changing trend of the cement concrete and asphalt concrete remains generally the same. When the ice thickness is fixed, as is shown 


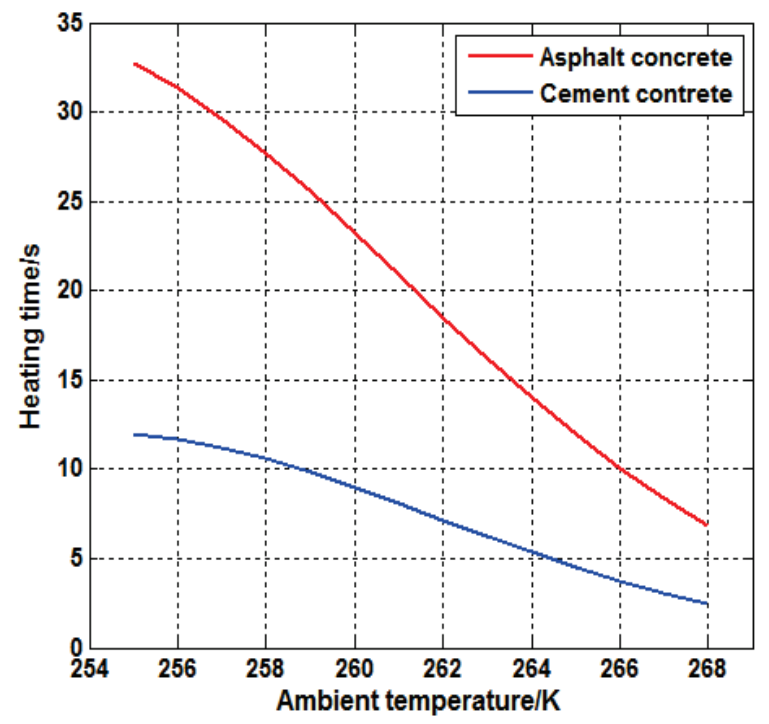

(a) The pavement material influence of different ambient temperatures

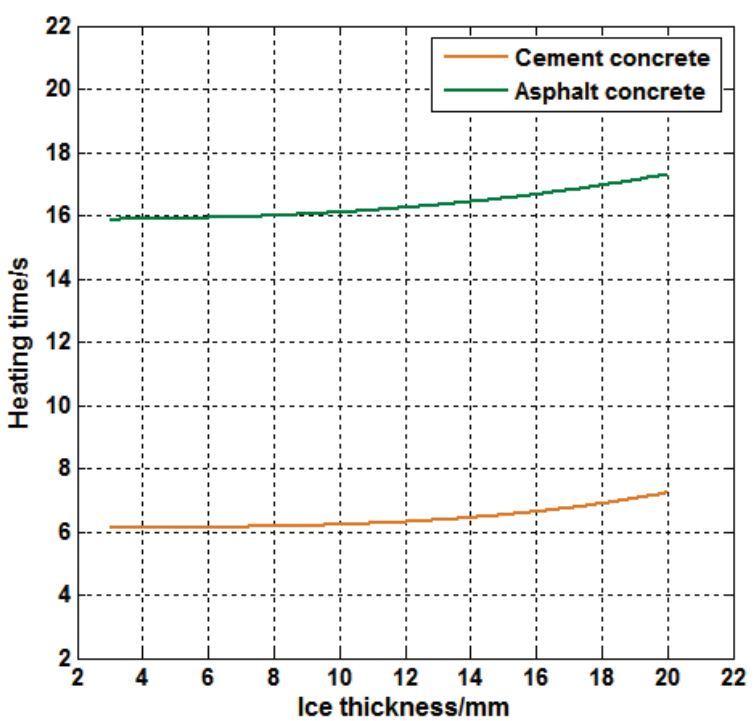

(b) The pavement material influence of different ice thicknesses

Fig. 8. (Color online) The influence of the pavement material.

in Fig. 8(b), the heating time of the asphalt concrete is roughly as three times as that of the cement concrete. Thus, it is not difficult to draw that the ice melting efficiency of cement concrete is higher than that of asphalt concrete, being three times as much. 


\section{Performance of Deicing Vehicle}

Applying the MW deicing principle, the MW heater can be loaded on the automobile chassis, and other auxiliary devices on the deicing vehicle can be assembled. Meanwhile the deicing efficiency is directly related to the power density, the longitudinal dimension of the MW heating device and the diving speed. In addition to several key indicators of the deicing vehicle, such as working width and ice thickness, the deicing efficiency will be evaluated in two indexes, the deicing vehicle driving speed and the deicing area per hour. Referring to national standard for deicing efficiency of the People's Republic of China, the minimum deicing vehicle speed is $5 \mathrm{~km} / \mathrm{h}$. Therefore, the reference deicing vehicle speed is set at $5 \mathrm{~km} / \mathrm{h}$, the relationship between MW power density, ambient temperature, the ice thickness and the length of heating wall can be obtained. The heating wall length is related to the product of heating time and vehicle speed.

As is shown in Fig. 9, when the ambient temperature and MW power density are fixed, the length of deicing vehicle heating wall required increases with the growth of ice thickness. Besides, when the ice thickness is constant, with the ambient temperature rising, the required length of the heating wall also increases. Meanwhile, when the ambient temperature and the ice thickness are fixed, the required heating wall length decreases while the power density increases. Therefore, if any two of them are given, the optimal length of the deicing vehicle heating wall can be worked out.

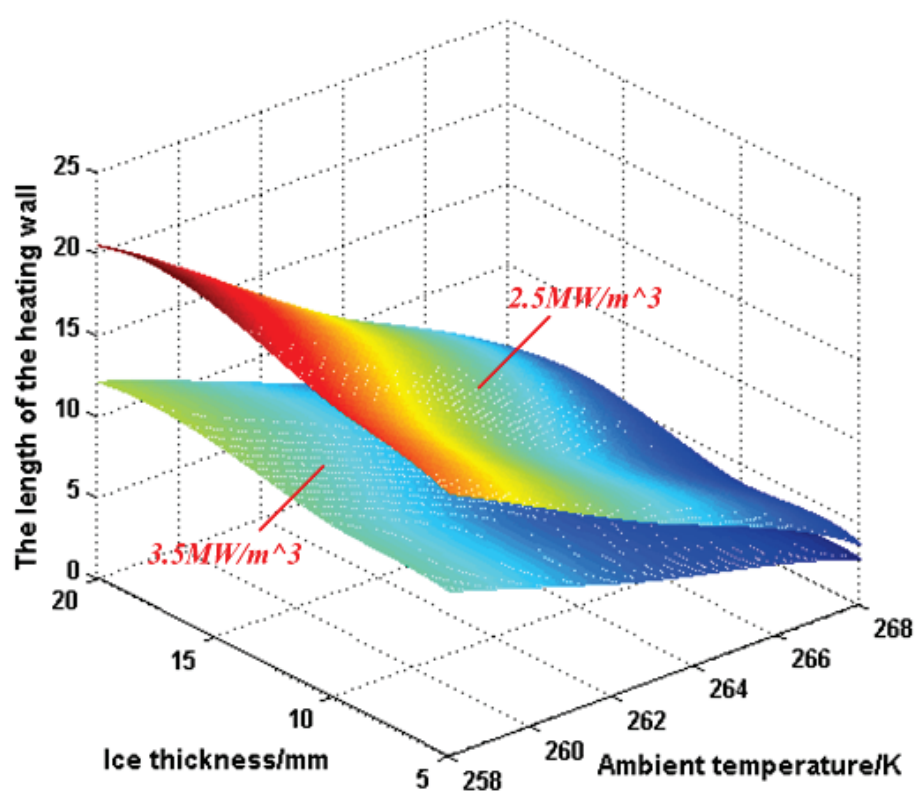

Fig. 9. (Color online) The relationship between the heating wall length and other factors. 
The sensibility of ice thickness is most significant, while the influence of ambient temperature is much smaller. Under some external conditions where ambient temperature is low and ice layer is thick, the length of heating wall or MW power may rise. However, if the ice layer is much thinner and the ambient temperature is lower, the heating power and wall length may not be enhanced. And either deicing efficiency or economy is the first demand for design, the optimal parameter can be comprehensively obtained.

\section{Conclusions}

Given the thermal variation and electrical properties of pavement materials, ice and water, a transient heat transfer model considering phase change of water and convection between ice surface and flowing air is employed in this paper. Based on the mathematical splitting operation with or without phase change heating, the finite differential method, assumptions about electromagnetic and heat transfer and analytic solutions is presented to describe the deicing process. The longitudinal temperature distributions of pavement and ice layer exposed to MW are developed in this time-dependent field, showing that the melting rate will increase to a great extent after appearing phase change.

With analysis of the influence factors on deicing efficiency through the characteristics of curve moving, the ice close to the ground melts first. For the ice thickness of $5 \mathrm{~mm}, 10 \mathrm{~mm}$ and $20 \mathrm{~mm}$, the shortest time of phase transitionis respectively $2.8 \mathrm{~s}, 6.05 \mathrm{~s}$ and $10.05 \mathrm{~s}$. In addition, the pavement material and ambient temperature influence the deicing efficiency more than the ice thickness, and the deicing efficiency of the road type of cement concrete is three times as rough asphalt concrete. The optimal economic and the deicing efficiency are the key to the deicing vehicle design, and if the environment conditions are given, such as ambient temperature and ice thickness, the optimal deicing vehicle heating wall and MW power can be approximately determined through this numerical result analysis.

\section{Acknowledgments}

The authors gratefully acknowledge the Hubei Key Laboratory of Advanced Technology for Automotive Components, Hubei Collaborative Innovation Centre for Automotive Components.

\section{References}

1. J. Jain, J. Olek, A. Janusz and D. Jozwiak-Niedzwiedzka, Transport. Res. Rec. 2290 (2012) 69-75.

2. Q. He, X. F. Lv and X. T. Zhao, J. Appl. Math. 2014 (2014) 8.

3. T. Yang, Z. Yang and M. Singla, J. Cold Regions Eng. 26(2) (2012) 55-70.

4. A. B. Crowley, Int. J. Heat Mass Transfer 21 (1978) 215.

5. J. Crank and R. S. Gupta, Int. J. Heat Mass Transfer 18 (1975) 1101. 
C. Wang et al.

6. P. Rattanadecho, Int. J. Heat Mass Transfer 47 (2004) 937-945.

7. B. J. Pangrle, K. G. Ayappa, E. Sutanto, H. T. Davis and E. A. Davis, Chem. Eng. Commun. 112 (1991) 39.

8. M. N. Ozisik, Heat Conduction, 2nd edn. (Wiley, New York, 1993).

9. T. Basak and K. G. Ayappa, AIChE J. 43 (1997) 1662-1674.

10. S. Bradshaw, S. Delport and E. Van Wyk, J. Microw. Power Electromagn. Energy 32 (1997) 88-95.

11. A. A. Salema and M. T. Afzal, Int. J. Therm. Sci. 91 (2015) 12-24.

12. H. Li and Q. Zhang, Cold Regions Sci. Technol. 103 (2014) 123-132.

13. D. E. Clark, D. C. Folz and J. K. West, Mater. Sci. Eng. A 287 (2000) 153-158.

14. M. Chamchong and A. K. Datta, J. Microw. Power Electromagn. Energy 34(1) (1999) $9-21$.

15. E. H. Andrews, H. A. Majid and N. A. Lockington, J. Mater. Sci. 19 (1984) 73-81.

16. GB/T 017-1996, The industry standard of the People's Republic of Chinese, Technical specification for construction of highway engineering JTJ 032 [s] (1996).

17. W. B. Fu and A. C. Metaxas, J. Microw. Power Electromagn. Energy 27(4) (1992) 217-222.

18. P. Ratanadecho, K. Aoki and M. Akahori, Appl. Math. Model. 26(3) (2002) 449-472.

19. D. D. Dincov, K. A. Parrot and K. A. Pericleous, J. Food Eng. 65 (2004) 403-412.

20. A. C. Metaxas and R. J. Meredith, Industrial Microwave Heating, IEEE Power Engineering Series, Vol. 4 (Peter Peregrinus, London, 1983).

21. K. Pitchai, S. L. Birla, J. Subbiah, D. Jones and H. Thippareddi, J. Food Eng. 112 (2012) 100-111.

22. K. G. Ayappa, H. T. Davis, G. Crapiste, E. A. Davis and J. Gordon, Chem. Eng. Sci. 46 (1991) 1005-1016.

23. S. A. Goldblith and D. I. C. Wang, Appl. Environ. Microbiol. 15(6) (1967) 1371.

24. R. Omar and A. Idris, Fuel 90 (2011) 1536-1544.

25. Y. Cheng, N. Sakay and T. Hanzawa, Food Sci. Technol. Int. 3(4) (1997) 324-328.

26. F. P. Incropera and D. P. DeWitt, Fundamental of Heat and Mass Transfer (John Wiley \& Sons, Inc., 2007).

27. L. Wang and X. Zhou, Heat Conduction (Springer-Verlag, Berlin, Heidelberg, 2008).

28. B. V. Karlekar and R. M. Desmond, Engineering Heat Transfer (West Publishing Company, St. Paul, MA, 1977).

29. Z. Wen, H. Chen and H. Dai, Heat Transfer (Shanghai Jiaotong University Press, Shanghai, China, 1987).

30. C. Eduardo, Heat Transfer in Process Engineering (McGraw-Hill, New York, 2010), pp. $43-44$.

31. X. Hu, H. A. Buckmaster and O. Barajas, Chem. Eng. Data 39 (1994) 625-635.

32. H. H. C. Jellinek, J. Colloid Sci. 14 (1959) 268-280. 